\title{
La diversification de la recherche en éthique animale et en études animales
}

\author{
RALPH R. ACAMPORA
}

Traduction par FRÉDÉRIC BAITINGER ${ }^{1}$

J'enseigne régulièrement des cours dans une variété de domaines que l'on regroupe généralement sous le terme d' «éthique appliquée», dont fait partie la recherche en éthique animale. Il n'y a pas si longtemps encore (il y a peut-être 10 ans), il n'existait aucun livre de référence sur le sujet. Pour constituer un plan de cours digne de ce nom, un professeur devait inclure dans son syllabus des monographies ou assembler une série d'articles ou d'essais provenant de sources différentes. À ma connaissance, le premier livre de référence dans ce domaine est paru au tout début de ce siècle (Hursthouse). En 2003 sont ensuite parus simultanément une anthologie pédagogique couvrant l'ensemble de l'éthique animale (Armstrong et Botzler), ainsi qu'une introduction générale (Taylor) : la première en est à sa seconde édition, tandis que le livre de Taylor en est déjà à sa troisième et a maintenant un nouveau concurrent (Gruen $)^{2}$.

Dans la plupart des cas, ces manuels ont tendance à couvrir le champ des recherches menées en éthique animale par la première génération de chercheurs spécialisés dans ce domaine. Cela signifie que ces manuels accordent la plus grande part de leur attention aux questions liées à la libération des animaux et aux théories des droits des animaux qui leur sont associées (je fais référence, ici, à la théorie utilitariste de Peter Singer et à la théorie déontologique de Tom Regan). 
Hursthouse et Gruen ont mis respectivement en lumière, dans leur livre, l'éthique des vertus et l'éthique féministe fondée sur le souci [care] pour autrui. Dans le même temps, les approches européennes et continentales ne semblent avoir reçu que peu d'attention. Taylor, comme Armstrong et Botzler, ont pourtant fait de leur mieux pour couvrir de manière large et plurielle l'ensemble des recherches effectuées en ce domaine — incluant dans leurs réflexions sur les matériaux empruntés à la phénoménologie, au pragmatisme et aux études sur les systèmes et processus. Néanmoins, ces approches ne sont généralement abordées que d'une manière relativement superficielle, pour ne rien dire des approches existentialistes, herméneutiques et postmodernes qui ne sont, quant à elles, évoquées qu'en passant, quand elles ne sont pas carrément passées sous silence. La situation que je viens d'esquisser est à peine différente quand on s'intéresse aux travaux plus généraux censés présenter l'état actuel de la recherche dans ce domaine ${ }^{3}$. Pourquoi une telle situation? Voilà une question que je me pose régulièrement.

\section{D’autres registres de moralité interspécifique}

À l'évidence, une part du problème peut être rattachée au jugement négatif (et parfois même rigide et obtus) que la philosophie anglo-saxonne continue à porter sur la philosophie continentale. Toutefois, une telle explication — compte tenu des rapprochements qui ont eu lieu ces derniers temps entre les deux camps — n'est ni suffisante ni, probablement, la plus importante. Une autre manière d'aborder la question m'est apparue récemment, et j'aimerais la formuler ici. Si les approches européennes en éthique animale ne reçoivent aucun écho favorable dans le monde universitaire anglo-saxon, cela, à mon avis, est dû au fait que les professeurs et chercheurs anglo-saxons ne cessent de commettre une erreur de jugement quant au type d'éthique 
que produit et approfondit la pensée continentale. Plus précisément encore, il me semble que les réflexions conduites par la pensée occidentale autour des questions morales interspécifiques ne sont pas tant effectuées du point de vue d'une éthique normative, que du point de vue de ce que la pensée anglo-saxonne appelle (de manière appropriée) la psychologie morale ou (d'une manière plus large) la méta-éthique ${ }^{4}$.

Une telle confusion quant à l'objectif que se donne la philosophie morale continentale (dès l'instant où elle entend traiter la question de l'éthique animale) pourrait expliquer pourquoi la pensée anglo-saxonne, de manière quelque peu dédaigneuse, a jusqu'ici jugé les apports de la philosophie continentale au domaine de l'éthique animale comme étant quasi inexistants. Car il est bien évident que si l'on s'attend à trouver dans des réflexions de type existentialiste, phénoménologique ou herméneutique sur la moralité interspécifique, les linéaments d'une éthique normative fondée sur des principes moraux susceptibles d'être convertis en prescriptions pratiques, on se condamne à être déçu ${ }^{5}$. En effet, les approches continentales, de par leur nature même, ne peuvent fournir d'éclaircissements qu'en ce qui a trait à la nature des comportements et des attitudes intentionnelles présupposées par les schémas d'éthique normative, ainsi qu'aux attitudes dispositionnelles (et intentionnelles) impliquées par de tels schémas ou inclus en eux de manière logique ( $c f$. Atterton et Calarco; et Steeves, Animal Others).

Il y a déjà eu un grand nombre d'interventions continentales dans un domaine qui pourrait être appelé la moralité «transhumaine» (qui ne doit pas être confondue avec le mouvement futuriste du transhumanisme). H. Peter Steeves, il y a quelques années déjà, a appliqué la phénoménologie husserlienne à une pensée communautariste teintée d'écologie ( $c f$. Steeves «Boudaries » et Founding Community). David Abram, dans une veine similaire, a fait usage de la pensée de Merleau-Ponty. Et enfin, Elizabeth Behnke, poursuivant un effort comparable, a 
développé une éthique [ethos] de la paix entre espèces à partir des œuvres des plus grands penseurs du courant phénoménologique. De manière plus récente, plusieurs théoriciens ont tenté de croiser de manière productive les résultats obtenus en éthique animale et en morale interspécifique, et les apports des philosophies existentialistes, herméneutiques et postmodernes de penseurs tels que Heidegger, Levinas, Derrida, Foucault ou Irigaray, pour ne citer que quelques noms (Atterton et Calarco). Je vais maintenant exposer ma propre contribution aux développements récents (d'orientation européenne) en éthique animale, après quoi je mettrai en lumière un motif important qui me semble prédominer dans tous ces travaux (y compris les miens).

Ma proposition revient à dire, en bref $^{6}$, que les chercheurs en éthique animale doivent éradiquer toute hiérarchie anthropologique - et cela, jusqu'au risque même de son émergence — pour hisser leur pensée morale et leur activisme politique au-delà d'une position posthumaniste type, position qui vise à réévaluer la notion d'animation corporelle en tant que telle. Car ce n'est qu'à travers la reconnaissance de notre propre statut d'animal vivant et zoomorphe qu'il devient possible, en retour, d'accroître l'étendue de notre souci de l'autre [caring regard]. Pour ce faire, il faut pouvoir ancrer notre compassion morale pour les animaux dans une sensation partagée de vulnérabilité charnelle (plutôt que de le faire à partir d'une position humaniste classique qui fait toujours de la mortalité un pur phénomène mental).

Si l'idée d'un tel «partage» de sensibilité est correctement expliquée, les liens qui existent entre morale et compassion pourront apparaître, à leur tour, de manière évidente. À un niveau purement somatique, par exemple, il est possible d'avancer l'idée que nous sommes conscients de notre propre vulnérabilité - aux blessures, aux maladies - en vertu du fait que nous sommes des êtres de chair et que nous partageons ce type de sensibilité somatique avec 
d'autres organismes, au sens où notre susceptibilité à la douleur est équivalente à celles des autres organismes vivants. Partant d'une telle proposition, ma thèse revient à dire que ce rapport minimal commun (fondé sur une même nature charnelle) est suffisant, d'un point de vue phénoménologique, pour établir un rapport compatissant à l'autre - pour autant que ce rapport puisse devenir l'objet adéquat de considérations éthiques. Dans un sens plus fort encore du mot «partage », il me semble que l'autre de la relation éthique [the second party], pour autant qu'il puisse aussi, parfois, être capable de prendre conscience de la vulnérabilité de celui qui lui fait face (dans la mesure où cette vulnérabilité est similaire à la sienne), pourrait former le socle d'une réciprocité enrichie, laquelle est nécessaire pour penser une forme de compassion réciproque comme le respect, c'est-à-dire une forme de relation dans laquelle chaque membre est capable de reconnaître l'autre en tant que sujet moral (autrement dit, en tant que sujet doué d'initiative $)^{7}$. Nos relations à certains primates (comme les hominidés) ou cétacés (comme les dauphins) et, plus encore, à l'ensemble des animaux domestiques ou de somme (comme les chiens ou les chevaux) ne contiennent-elles pas, d'ailleurs, des éléments de compassion conscients et réciproques qui se sont développés jusqu'à prendre la forme d'un véritable sentiment de respect ${ }^{8}$ ?

Quelle signification éthique peut bien avoir le développement d'une somatologie interspécifique? Je soutiens l'idée qu'une telle recherche fournit la clé expérimentale nécessaire à l'étude de ce qu'on nomme généralement le «sens moral» — thèse qui prend tout son relief quand on l'applique à l'étude de cas interspécifiques. En ce sens, d'ailleurs, mon combat a toujours été de dire (et surtout dans les cas trans-spécifiques) que la vie morale, de manière primordiale, est enracinée — phénoménologiquement — dans une corporéité symphyse plutôt que dans des processus mentaux gouvernés par une conception standard de la sympathie. Tel que 
je l'utilise, le terme «symphyse » désigne le fait, pour un être, de sentir qu'il partage avec un autre être un lien vital, lien dont il fait l'expérience à travers une série de dispositions somatiques, directes ou systémiques, (inter)relationnelles ${ }^{9}$. Ma position revient donc à dire que parler de symphyse est le meilleur moyen de décrire le sentiment (proto éthique) qui nous prépare à tenir compte des autres animaux à l'intérieur de nos jugements éthiques ${ }^{10}$. Les raisonnements inférentiels par analogie pourraient venir justifier rationnellement mon hypothèse, et la psychologie de l'imagination pourrait scientifiquement l'expliquer à travers l'étude des phénomènes de projection empathique. Mais seule la «somatologie » d'être d'une même espèce, ou possédant des attributs spécifiques, peut convenablement (et phénoménologiquement) articuler l'expérience actuelle d'une telle co-appartenance ${ }^{11}$. À partir de là, et ultimement, je dirais que seule une éthique sculptée à partir des interactions symphyses est capable d'engendrer un caractère ou une culture moralement attentifs à leur élément existentiel, c'est-à-dire à leur être-au-monde charnel — être-au-monde qui inclut la vulnérabilité charnelle que nous partageons avec tous les corps vivants en tant que tels ( $c f$. Cataldi, Sullivan et Shusterman).

La perspective que je viens d'esquisser recoupe plusieurs réflexions faites par la pensée continentale en matière d'éthique animale : l'idée, par exemple, que l'attention donnée à une visée morale ne peut être fondée que sur l'affectivité et la réceptivité d'un sujet moral; ou encore l'idée que la vulnérabilité ou la mise à nu de notre capacité à être touchés moralement — dans des expériences de compassion ou d'empathie - est une expérience qui permet d'unir l'idée de visée morale à celle de réceptivité charnelle. Par-delà ces éléments, ma position partage avec l'approche européenne de la morale interspécifique certains éléments méthodologiques — ces éléments tendant toujours à reconfigurer notre compréhension de la psychologie morale d'une manière qui n'est pas sans rappeler ce qui se produit en éthique animale quand on passe d'un 
modèle fondé sur des droits rationnels et des schémas de calcul d'utilité, à un modèle fondé sur l'idée de ressemblance substantielle entre espèces. Les approches traditionnelles de la psychologie morale peuvent être réduites, en effet, à l'expression de deux tendances: la première, centrée sur l'éthique normative, se concentre sur la déontologie, l'utilitarisme ou l'éthique de la vertu; la deuxième, centrée sur la psychologie, s'appuie sur les approches cognitivistes et behavioristes ou biomédicales ( $c f$. Sinnott-Armstrong). Or, ce que représente, pour moi, l'approche continentale de la psychologie morale diffère justement de ces deux tendances, dans la mesure où (1) elle mobilise ou admet des vues éthiques hétérodoxes fondées sur la sensibilité ou le souci de l'autre, et (2) elle tend à invoquer des (ou à dépendre de) courants de pensées psychologiques qui n'appartiennent pas au courant universitaire dominant (je pense ici à des courants existentialo-humanistes, phénoménologico-herméneutiques ou néo et postfreudiens). Mon propre projet, que j'appelle somatologie, se fonde sur une approche husserlienne de la conscience incarnée. Il se situe quelque part entre les approches traditionnelles de la psychologie et de la physiologie. Son ambition est de développer ce que j'appelle une «physique du senti»des organismes animés. De là mes efforts pour dessiner la texture psychosomatique des expériences morales interspécifiques (Corporeal Compassion ch. 4).

\section{L'ontologie revisitée : animalité et altérité}

J'aimerais maintenant élargir mon champ d'analyse afin d'y inclure une vue d'ensemble des études animales. J'analyserai d'abord les changements imminents qui s'apprêtent à se produire dans ce domaine, puis je m'efforcerai de relier ces changements aux développements précédemment analysés. Pour commencer, je voudrais rappeler les principaux courants qui 
animent les études animales depuis leur création en 1990, et souligner le fait que ce domaine s'est constitué à partir d'un certain nombre de prises de positions ontologiques - lesquelles s'enracinent elles-mêmes dans les ambiguïtés multiples que comporte le mot «animal». Ce terme peut en effet être utilisé pour faire référence à tout type d'espèce ou de spécimen animal, ainsi que pour faire référence à toutes les espèces animales non humaines. Mais il peut aussi faire référence, de manière interne, à certains aspects (biologiques ou émotionnels) de notre propre condition (généralement humaine). Les chercheurs en études animales, empruntant à Hegel le dynamisme de ses analyses (l'idée du devenir autre de la conscience de soi), mais refusant l'idée de totalité synthétique, ont jusqu'ici eu bien du mal à démontrer le caractère glissant de catégories abstraites d'humanité ou d'animalité (en tant que terme abordé d'une manière purement externe). Ces deux dernières décennies, de nombreux penseurs - comme l'écologiste Paul Shepard (Others), l'anthropologue Deborah Bird Rose, la philosophe Kelly Oliver ou le théoricien littéraire Cary Wolfe, pour n'en nommer que quelques-uns — ont déconstruit l'idée d'identité humaine. L'effet cumulatif de leurs travaux aura permis de démontrer à quel point l'idée d'humanité est fondée, profondément et perversement, sur un processus d'abjection des éléments autres que sa représentation exclue — ces éléments formant, le plus souvent, ce qui a trait à la notion d'animalité (les instincts, les pulsions, mais aussi certains processus et attitudes cognitifs) et faisant pourtant partie intégrante de la construction que l'on appelle, de façon bien trop simpliste, l'humanité.

D'une manière similaire, une autre série d'efforts accomplis en études animales a permis de diversifier les approches de la notion abstraite d'animalité, approches qui, jusque-là, s'étaient contentées d'aborder la notion d'animalité de façon extérieure. À l'instar des terminologies génériques qui servirent naguère à définir les idées de race et d'ethnie ou des catégories 
démographiques telles que « Nègre », « Juif » ou « Orient(al) — — catégories qui sont tombées en désuétude après avoir fait l'objet de critiques décapantes de la part d'un grand nombre de chercheurs en sciences sociales —, les chercheurs en études animales se sont efforcés de rendre apparente la logique d'effacement de la différence sur laquelle se fonde et se perpétue la myopie d'un terme comme celui d'« animalité ». Élaborant leurs discours à partir de celui de la biologie et de la zoologie (et, notamment, à partir des œuvres des figures fondatrices de ces deux disciplines, de Darwin à Hediger), les chercheurs en études animales sont parvenus à éroder conceptuellement les stéréotypes sur lesquels était fondé leur propre domaine d'étude. Ce mouvement critique, sans aucun doute, a atteint un sommet avec la conférence donnée par Derrida à Cerisy en 1997, «L'animal que donc je suis... ». Cette conférence souvent citée, dans laquelle il se livre à une opération de déconstruction du terme «animot », accomplit, pour le domaine des études animales, ce qu'Edward Saïd opéra dans le domaine des sciences sociales avec son livre L'orientalisme. Ce processus déconstructif a permis de recentrer l'attention des chercheurs sur la spécificité (et, quelque fois même, sur l'individualité) de l'être animal et de son devenir - parler de tel cheval, de tel corbeau, de telle pieuvre, plutôt que du cheval, du corbeau ou de la pieuvre en général - et, inversement, de mettre un terme aux réflexions sur ce qu'est «l'animalité » en tant que telle ou sur ce que sont les animaux en général (généralités qui invitent à des dérapages idéologiques dans la mesure même où leurs références ne sont jamais qu'abstraites ou indéterminées, voire, dans le pire des cas, purement inexistantes).

Jusqu'à présent, les études animales sont parvenues à mettre en évidence la manière dont le «jeu de la différence » traverse toute définition de la nature humaine et, réciproquement, toute définition du règne animal (en tant qu'il s'oppose au règne humain). La nouvelle tâche qui commence à poindre à l'horizon des études animales devrait donc se confondre avec celle de 
déconstruire l'essence de l'animalité en tant que telle, en prenant pour point de départ ses propriétés internes. Déjà suggéré par le dernier ouvrage de Wolfe, What is Posthumanism?, ainsi que par une série d'articles et d'essais parus (ou sur le point de paraître) dans divers journaux scientifiques (comme Antennce ou Social Text), cette opération critique — à travers laquelle le champ des études animales ose enfin s'attaquer au concept qui le fonde - me semble être une preuve de maturité. Les expériences que l'on peut faire de l'animalité et les idées à ce sujet se superposent souvent à des zones ou à des axes d'altérité, à travers lesquels ce qu'on appelle «animal » peut en venir à être pensé et senti comme exhibant des traces, ou des forces, de son autre — tout comme il a été démontré que l'humanité n'est (et ne devient) qu'en tant qu'elle est le lieu d'une différence. Prenons, comme exemple insigne, le concept clé d'«être animé » : ce concept, à l'évidence, est au cœur du concept d'animalité. Pourtant, il semble déjà préfiguré dans les modes de développement des plantes ou des microbes, et il résonne aussi dans les mouvements mécaniques des machines ou des codes. Attribuer de la vitalité au règne végétal, aux bactéries, à la prolifération cellulaire dans le plasma, dément la présomption selon laquelle seule la faune capable de se déplacer sur une échelle spatio-temporelle ajustée à la nôtre posséderait, d'un point de vue molaire, un principe d'animation $(c f \text {. Marder })^{12}$. De la même manière, les développements récents en biorobotique, ainsi que les expériences menées sur la vie et l'intelligence artificielles, rendent suspecte la présupposition selon laquelle la faculté d'animation, ou d'être doué de vie, devrait nécessairement reposer sur une base organique. Inversement, et de manière intéressante pour mon propos, si l'on juxtapose l'héliotropisme des plantes, des arbres, des tortues, des lézards, à l'inclination toute humaine à aller s'étendre sur une plage, ou bien encore, quand on tient compte des symbioses microbiennes, de la double hélice ADN/ARN et des fantasmagories autour des cyborgs ( $c f$. Haraway et Mazis), force est de 
reconnaître que nous avons incorporé, dans la conception que nous nous faisons de l'animal en tant qu'autre, une part de nous-mêmes.

Pour certains, je le conçois, mon chemin de pensée peut paraître un peu désuet. Et il qu'Aristote avait déjà développé dans De Anima toutes les imbrications du concept d'animation dans sa théorie philosophique et biologique de l'âme. En effet, les plantes, pour le Stagirite, possédaient une âme végétative et étaient dotées de deux mouvements : celui de la nutrition et de la génération. Les êtres vivants non humains, qui possédaient une âme motrice, étaient selon lui dotés de deux autres mouvements : la locomotion et l'appétit (le désir vital). Les êtres humains, enfin, qui possédaient une âme intellective, étaient dotés de la capacité de connaître le monde de manière rationnelle. De plus, chaque niveau d'animation était conçu, chez Aristote, comme incorporant (plutôt que comme les englobant), dans son principe, les niveaux inférieurs. Et c'est ainsi que pendant des siècles, du Moyen Âge jusqu'à l'époque moderne, la doctrine chrétienne de la scala naturce a pu lentement prendre forme et s'imposer. La filiation de cette «grande chaîne des êtres » a été reconstituée d'une manière extrêmement convaincante par Arthur Lovejoy qui a réaffirmé la pertinence d'une telle idée pour penser, de manière générale, les liens qui unissent tous les niveaux et tous les êtres de la création - et cela, bien avant les avancées contemporaines en études animales. Cependant, ce schéma, tout comme celui d'Aristote, ne possède au mieux que des ressemblances superficielles avec le type de tissage organique qui est en voie d'être théorisé dans le domaine des études animales. Les deux vieilles doctrines de l'âme consistent essentiellement, il est vrai, en une répartition hiérarchique des êtres, laquelle représente justement l'idée la plus contestée par les chercheurs en études animales (ou du moins, par les chercheurs d'avant-garde). 
Alors qu'Aristote concevait le monde biologique comme une imbrication d'âmes différentes (et dont le sommet coïncidait avec l'idée qu'il se faisait de l'homme), la théoricienne posthumaniste Rosi Braidotti pose à l'origine du monde biologique un principe dynamique amorphe et exubérant : «Le couple bios/zoé, en tant que principe génératif et vital, est une force majeure qui traverse et relie des domaines qui, jusque-là, étaient restés séparés » (Braidotti 99). Nulle chaîne ici, moins encore d'êtres, mais plutôt une interprétation fluide de zones douées de mouvements, dont les axes sont constamment soumis à des changements et à des réorientations ${ }^{13}$. Dans une veine similaire, l'écocritique développée par Timothy Morton est devenue, sur le tard, le péan de l'hybridité : «Tous les organismes sont des monstres, dans la mesure où ils ne sont que des chimères composées de parties empruntées à d'autres créatures » (Morton 66). De merveilleux «étranges étrangers », des formes vivantes passant les unes dans les autres : « Ce qu'il y a de dérangeant à propos des animaux », suggère Morton, «c'est qu'au fond [au niveau des algorithmes biochimiques], ce sont des plantes » (68). Ce penchant des processus vitaux à se répéter sous une forme récursive permet de relier le monde animé aux éléments inorganiques qui le composent; en d'autres termes, et pour m'aventurer jusqu'aux origines de la génétique, « le mouvement qui a commencé la vie est à trouver dans la matière elle-même»(67). C'est pourquoi, à suivre les raisonnements des penseurs que je viens de citer (et qui forment l'avantgarde des études animales), la valse de l'identité et de l'altérité doit être conçue comme une danse qui traverse de part en part la notion d'animalité et, par ricochet, la notion de son autre (c'est-à-dire de ce qui s'oppose à elle).

Pour conclure ma réflexion, $\mathrm{j}$ 'aimerais récapituler, et penser ensemble les points essentiels de mon propos, et, d'une manière plus ambitieuse, mettre en lumière l'une des implications importantes qui semble émerger d'une telle mise en relation. Au cours de mes réflexions sur 
l'éthique animale, j'ai avancé l'idée selon laquelle les contributions les plus récentes qui ont été faites dans le domaine par la dernière génération de chercheurs ont toutes été réalisées à partir d'une approche philosophique de type européenne et continentale, c'est-à-dire à partir d'un point de vue qui n'est pas celui de l'éthique normative à proprement parler, mais qui devrait plutôt être qualifié comme étant celui de la méta-éthique ou de la psychologie morale. Ce qu'une telle approche permet de découvrir et de mettre en lumière, c'est l'importance primordiale que jouent, dans le domaine de la moralité interspécifique, les émotions et les formes incarnées de contact à l'autre (à travers, par exemple, les expériences affectives, somatiques, ou bien encore à travers les expériences concrètes que l'on peut faire de sa propre vulnérabilité). Or, à n'en pas douter, une telle approche s'écarte beaucoup des analyses anglo-saxonnes qui ont été produites par la première génération de chercheurs en éthique animale — analyses reconnues pour mettre de l'avant les idées de sapience ou de sentience (en tant que ces notions permettent de penser la subjectivité), de capacité à produire des règles ou à faire des choix rationnels (fondés sur l'idée de calcul de l'utilité) ou à tenir des discours abstraits.

Considérer un domaine comme celui de l'éthique animale à partir d'un point de vue analytique revient presque toujours à prendre (explicitement ou implicitement) l'«esprit humain » comme paradigme de toutes les autres formes de capacité éthique et, par-là même, à faire de l'idée de mentalité la pierre de touche à partir de laquelle il devient possible de construire une vision morale - vision qui, conséquemment, s'appuie largement sur les recherches en neurosciences et en sciences cognitives afin de prouver qu'il existe bien, chez les animaux non humains, des éléments comparables à ceux que l'on trouve dans la pensée humaine. Ces recherches restent, en un certain sens, utiles, pour autant qu'elles aident à lutter contre ce que le primatologue Frans de Waal appelle «le déni anthropologique» (Waal 50-53). Toutefois, un tel 
déni possède un envers, à savoir le refus de percevoir dans notre propre être des attributs zoomorphes. C'est pourquoi la subversion d'un tel déni requiert, à mon sens, de pouvoir adopter un autre point de vue sur la question, point de vue que la philosophie continentale (la philosophie européenne, mais aussi la philosophie féministe fondée sur le care) peut seule nous donner. Dans la mesure, en effet, où les générations les plus récentes de chercheurs en éthique animale ont pris comme point de départ de leurs réflexions un cadre théorique hétérodoxe (cadre qui les plonge d'emblée dans l'expérience sensible d'une conscience incarnée), ceux-ci ont enfin pu devenir sensibles à toutes les forces et faiblesses qu'englobe le champ de la vie (au lieu de n'être fascinés que par la splendeur de notre propre espèce et de chercher dans les autres espèces animales des éléments qui pourraient les rapprocher de nous). C'est à partir d'une telle ouverture que mes propres réflexions - qui mettent en jeu les notions d'(inter)relations, de superpositions et de croisements — doivent être abordées.

Autrement dit, la diversification des recherches en éthique animale et en études animales peut être considérée, d'un point de vue global, comme un tissage qui modifie nos conceptions de la morale et de l'animalité tout en les enrichissant. L'alternative, ou plutôt: le changement d'approche que je viens de définir est une tentative pour dépasser (transcender), d'une manière plus rigoureuse que ne l'ont fait les études passées, le point de vue anthropocentrique qui prévalait jusqu'ici dans ce domaine d'étude — approche qui, à première vue, requiert une conception polycentrique de l'épistémologie et de l'éthique. Toutefois, une telle idée doit ellemême faire l'objet d'une correction, dans la mesure où, dans chaque opération menée dans ce champ, la multiplicité des points de vue mis en jeu aboutit à la destruction de l'idée de centre. C'est pourquoi, plutôt que de parler de polycentrisme, il me semble préférable de parler « d'acentrisme» (cf. Weston). Néanmoins, cela n'implique pas que nous serions pris dans un jeu 
de différences entropique et sans fin, mais plutôt que nous devons faire plus attention au caractère affectif et incarné de toute expérience éthique, ainsi qu'aux stries (ou au scories) d'altérité qui, ontologiquement, nous impliquent (nous, en tant qu'êtres humains) dans la tension qui existe entre l'idée de similitude et celle de variété ( $c f$. Aaltola). La fascination qu'engendre une telle tension, tout autant que la faculté de la mesurer, semble être précisément ce qui, dans l'étude des autres animaux, est à la fois attirant et contraignant : à partir d'une telle perspective, en effet, de nombreuses créatures deviennent pour nous ni des occurrences d'une similitude déviante (comme c'est le cas dans une perspective anthropocentrique) ni des exemples d'une altérité radicale (comme c'est le cas dans une perspective poststructuraliste), mais plutôt quelque chose comme des instances d'altérité relative (comme c'est la cas dans les recherches séminales, pour ne pas dire déviantes, que mène le chercheur en études animale Paul Shepard [Thinking Animals]).

\section{Notes}

1. Traduction revue par Martine Béland. Une partie de ce texte figure dans un chapitre sur les approches continentales en philosophie animale, à paraître en 2014 (en version électronique) et 2015 (en version imprimée) dans le Handbook for Animal Studies (Oxford University Press).

2. Telle est l'évolution des publications en langue anglaise; mes compétences en langues étrangères ne me permettent malheureusement pas de comparer cette évolution à celle qui a lieu dans d'autres langues.

3. Et cela, même dans une anthologie dédiée à l'exégèse continentale, comme celle d'Atterton et Calarco. Il est intéressant, par ailleurs, de noter (d'une manière ironique ou emblématique) à quel point Peter Singer, dans son avant-propos, critique et dévalorise les traditions continentales.

4. Quelques précisions terminologiques : en philosophie contemporaine, le domaine de la métaéthique renvoie à des recherches très abstraites ou bien à des recherches d'un second ordre touchant aux fondements des théories et des codes moraux en tant que tels (l'enjeu, ici, n'étant 
plus de pouvoir définir ce que chacun doit faire, mais ce que veut dire l'idée même de devoir : autrement dit, le problème n'est plus de savoir ce qui est juste ou bon, mauvais ou diabolique, mais de savoir si des notions telles que celles de bien et de mal existent réellement). Le domaine de la «psychologie morale » (parfois conçu comme une partie des recherches en méta-éthique, parfois comme un domaine de recherche interdisciplinaire autonome) renvoie à une constellation d'études qui cherchent à définir quelles sont les conditions (ou les prérequis) nécessaires pour pouvoir se comporter (im)moralement ou (an)éthiquement.

5. Bien évidemment, de tels éléments ne sont pas l'apanage exclusif des approches continentales (que ce soit le souci de l'autre ou le souci écologique). Les approches pragmatistes, processuelles ou féministes peuvent aussi être pertinentes en psychologie morale - c'est pourquoi la validité de mon explication peut paraître ici un peu forcée. Trois précisions, toutefois, peuvent clarifier la situation. Premièrement, les féministes et les philosophes de tradition américaine ont su mieux définir et délimiter leurs contributions au domaine de la moralité interspécifique que les philosophes travaillant dans la tradition européenne; deuxièmement, les chercheurs appartenant à la tradition américaine se sont montrés plus enclins à confronter les résultats de leurs recherches avec ceux obtenus dans les domaines de l'éthique normative et de l'éthique appliquée; ces chercheurs ont aussi été les auteurs ou les éditeurs d'anthologies (alors que les chercheurs européens n'ont pas publié de telles anthologies - tout au moins des anthologies de type pédagogique). C'est pourquoi, au final, ils se sont retrouvés dans une meilleure position pour exposer clairement les raisons de leurs préférences méthodologiques.

6. Dans le passage qui suit, je réécris, sous une forme condensée, certaines réflexions exposées dans mon « Somatic Sympathy .

7. Les facteurs mentaux (comme la conscience de second ordre dans les processus de reconnaissance mutuelle) doivent aussi intervenir pour que la notion de respect, telle que je viens de la définir, puisse véritablement émerger en tant que phénomène moral.

8. Lors de la pré-conférence d'un symposium qui s'est tenu juste avant le premier congrès de Minding Animals, Gruen a justement indiqué que cet argument n'a qu'une portée limitée aux chimpanzés (communication orale).

9. Seamon utilise ce terme dans son chapitre «Different Worlds Coming Together ». Ce terme, comme le note justement Seamon, tire son origine d'un usage médical et signifie, en grec ancien, «le fait de grandir ensemble " [《the state of growing together »] (Seamon 23). Son équivalant latin pourrait être trouvé dans le terme "concressence», mais ce terme - au moins dans le discours philosophique - a acquis une connotation whiteheadienne qui n'est pas forcément adéquate à l'usage que je voudrais en faire. Il est aussi important de noter que la symphyse n'est pas un état de fusion totale : comme le stipule Kuang-Ming $\mathrm{Wu}$, "le "couple" s'interpénètre en osmose, s'infuse mutuellement mais, en même temps, conserve l'intégrité de ses parties » (Wu 12 , je souligne). 
10. Hacking semble lui aussi suggérer qu'il existe un mode de conscience symphysique lorsqu'il dresse le portrait d'une expérience morale - expérience «qui est une relation fondée sur une identité corporelle commune. C'est une relation de sympathie entre l'homme et la bête [sic], qui fonctionne à partir du corps vivant des deux » (Hacking 691, je souligne).

11. La philosophie gréco-germanique, d'Aristote à Hegel et Marx, s'est développée à partir d'une tradition anthropologico-philosophique fondée sur l'étude exclusive de l'être de l'espèce humaine; la tâche que se propose le transhumanisme est d'aller au-delà d'une telle tradition (strictement humaine), pour s'acheminer vers une ontologie générique de l'animalité (s'il peut y en avoir une) ou bien vers la formulation d'une ontologie des espèces autres qu'humaines.

12. Il est possible, aussi, de prendre comme exemple les espèces de mangroves australiennes qui se déplacent effectivement : quand bien même leurs déplacements, considérés de notre point de vue, sont lents, il n'en reste pas moins que ces plantes sont capables de transplanter leurs racines dès l'instant où la profondeur ou la salinité de l'eau changent!

13. Il y a des échos nietzschéens dans une telle assertion. $C f$. par ex. le fragment posthume 38[12] (juin-juil. 1885) : «Ce monde : un monstre de force, sans commencement ni fin [...] qui ne s'use pas mais se transforme [...] comme un jeu de forces et d'ondes de force, s'accumulant sur un point si elles diminuent sur un autre; une mer de forces en tempête et en flux perpétuel, éternellement en train de changer, éternellement en train de refluer » (Nietzsche 343 sq.).

\section{Textes cités}

Aaltola, Elisa, " "Other Animal Ethics" and the Demand for Difference », Environmental Values, vol. 11 (2002), p. 193-209.

ABrAm, David, The Spell of the Sensuous, New York, Random/Vintage, 1996/1997.

—, Becoming Animal, New York, Random/Vintage, 2010/2011.

ACAMPORA, Ralph R., Corporal Compassion. Animal Ethics and Philosophy of Body, Pittsburg, University of Pittsburg Press, 2006.

—, «Somatic Sympathy », in G. SMulewiCZ-ZuCKER (dir.), Strangers to Nature. Animal Lives and Human Ethics, Lanham, Lexington Books, 2012.

Antennae, numéro thématique : «Why Look at Plants? », vol. 17 (2011).

Armstrong, Susan et Richard Botzler (dir.), Animal Ethics Reader, Londres, Routledge, 2003.

Atterton, Peter et Matthew Calarco (dir.), Animal Philosophy. Ethics and Identity, Londres, Continuum, 2004. 
BEHNKE, Elizabeth, «From Merleau-Ponty's Concept of Nature to an Interspecies Practice of Peace », in H. P. SteEves (dir.), Animal Others. On Ethics, Ontology, and Animal Life, Albany, SUNY Press, 1999, p. 93-116.

BIRD Rose, Deborah, Dingo Makes Us Human. Life and Land in an Australian Aboriginal Culture, Cambridge, Cambridge University Press, 1992.

BraidotTi, Rosi, Transpositions. On Nomadic Ethics, Londres, Polity Press, 2006.

Cataldi, Susan, Emotion, Depth, and Flesh. A Study of Sensitive Space: Reflections on Merleau-Ponty's Philosophy of Embodiment, Albany, SUNY Press, 1993.

DARwIN, Charles, «On the Tendency of Species to Form Varieties », Londres, Linnean Society, $1^{\text {er }}$ juil. 1858 (discours).

DERRIDA, Jacques, L'animal que donc je suis, Paris, Galilée, 2006.

GRUEN, Lori, Ethics and Animals. An Introduction, Cambridge, Cambridge University Press, 2011.

HACKING, Ian, « On Sympathy : With Other Creatures », Tijdschrift voor Filosofie, $\mathrm{n}^{\circ} 63$ (2001), p. 685-717.

HARAWAY, Donna, Modest_Witness@Second_Millennium.FemaleMan_Meets_OncoMouse, New York, Routledge, 1997.

HEDIGER, Heinim, Wild Animals in Captivity, trad. G. Sircom, Londres, Butterworth, 1950.

Hursthouse, Rosalind, Ethics, Humans, and Other Animals. An Introduction with Readings, Londres, Routledge, 2000.

Lovejoy, Arthur, The Great Chain of Being. A Study in the History of an Idea, Cambridge, Harvard University Press, 1964 (1936).

MARDER, Michael, «Plant-Soul. The Elusive Meanings of Vegetative Life », Environmental Philosophy, vol. 8, n 1 (2011), p. 83-99.

MAZIs, Glen, Humans, Animals, Machines. Blurring Boundaries, Albany, SUNY Press, 2008.

MorTON, Timothy, The Ecological Thought, Cambridge, Harvard University Press, 2010.

NIETZSCHE, Friedrich, Euvres philosophiques complètes, éd. Colli et Montinari, vol. XI : Fragments posthumes (automne 1884 - automne 1885), trad. M. Haar et M. B. de Launay, Paris, Gallimard, 1982.

Oliver, Kelly, Animal Lessons. How They Teach Us to be Human, New York, Columbia University Press, 2009.

SEAmon, David, Dwelling, Seeing, and Designing. Toward a Phenomenological Ecology, Albany, SUNY Press, 1993.

SHEPARD, Paul, Thinking Animals, New York, Viking, 1978. 
—, The Others. How Animals Made Us Human, Washington/Covelo, Island Press, 1997.

Shusterman, Richard, Body Consciousness. A Philosophy of Mindfulness and Somaesthetics, Cambridge, Cambridge University Press, 2008.

SinnotT-ARMStrong, Walter (dir.), Moral Psychology, vol. 1-4, Cambridge, MIT Press, 20072014.

Social Text, numéro thématique : «Interspecies », vol. 106 (2011).

Steeves, H. Peter, «The Boundaries of the Phenomenological Community », in R. FISCHER (dir.), Becoming Persons, Oxford, Applied Theology Press, 1995, p. 777-797.

—, Founding Community, Dordrecht, Kluwer, 1998.

—, Animal Others. On Ethics, Ontology, and Animal Life, Albany, SUNY Press, 1999.

Sullivan, Shannon, Living Across and Through Skins. Transactional Bodies, Pragmatism, and Feminism, Bloomington, Indiana University Press, 2001.

TAYlor, Angus, Animals and Ethics. An Overview of the Philosophical Debate, Toronto, Broadview Press, 2003.

WAAL, Frans de, «Are We in Anthropodenial? », Discover, vol. 18, n 7 (1997), p. 50-53.

Weston, Anthony, « Multicentrism : A Manifesto », Environmental Ethics, vol. 26 (2004), p. 2540.

Wolfe, Cary, What is Posthumanism?, Minneapolis, University of Minnesota Press, 2010.

Wu, Kuang-Ming, On the Logic of Togetherness. A Cultural Hermeneutic, Leyde, Brill, 1998. 